\title{
Physical Activity and Self-Reported \\ Cardiovascular Comorbidities in Persons with Multiple Sclerosis: Evidence from a Cross-Sectional Analysis
}

\author{
Robert W. Motla Bo Fernhall ${ }^{\mathrm{a}}$ Edward McAuley ${ }^{\mathrm{a}} \quad$ Gary Cutter $^{\mathrm{b}}$

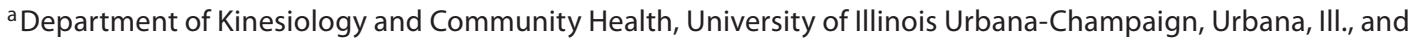 \\ ${ }^{b}$ Department of Biostatistics, University of Alabama, Birmingham, Ala., USA
}

\section{Key Words}

Cardiovascular comorbidities • Multiple sclerosis •

Physical activity

\begin{abstract}
Background: This study examined the possibility of a linear, inverse association between physical activity and the number of self-reported cardiovascular comorbidities in persons with multiple sclerosis (MS). Methods: The sample included 561 persons with MS who completed demographic, cardiovascular comorbidity, disability status, and physical activity self-report assessments, and then wore an accelerometer for 7 days. The data were analyzed using bivariate correlation and multiple linear regression analyses. Results: Bivariate correlation analysis indicated that there were statistically significant, inverse associations between the number of selfreported cardiovascular comorbidities and objectively measured $(r=-0.192, p=0.0001)$ and self-reported $(r=-0.151$, $p=0.0001)$ physical activity. The first multiple linear regression indicated that objectively measured physical activity was significantly associated with the number of self-reported cardiovascular comorbidities $(B=-0.003$, SE $B=0.001$, $\beta=-0.128$ ), even after controlling for confounding variables. The second multiple linear regression indicated that self-re-
\end{abstract}

ported physical activity, too, was significantly associated with the number of self-reported cardiovascular comorbidities $(B=-0.011$, SE $B=0.004, \beta=-0.114)$, even after controlling for confounding variables. Conclusion: Physical activity was associated with the number of self-reported cardiovascular comorbidities, independent of disability status and other possible confounding variables, in persons with MS.

Copyright $\odot 2011$ S. Karger AG, Basel

\section{Introduction}

Multiple sclerosis (MS) is a chronic, progressive disease of the central nervous system that affects an estimated 1 per 1,000 adults in the United States [1] and an estimated 2.5 million adults worldwide. Recent evidence indicates that persons with this neurological disease have increased risks of physical comorbidities (self-reported) [2] and cardiovascular comorbidities (cases) [3, 4]. Selfreported cardiovascular comorbidities have been associated with a more rapid progression of disability in MS [5]. Persons with MS further experience a shortened life span and, while younger persons with MS die due to complications of MS such as infections, cardiovascular causes do contribute to this shortened life span [6-8]. Physical in-

\section{KARGER}

(C) 2011 S. Karger AG, Basel

Fax +41613061234 E-Mail karger@karger.ch www.karger.com www.karger.com/ned
Robert W. Motl, PhD

Department of Kinesiology and Community Health

University of Illinois, 350 Freer Hall

Urbana, IL 61801 (USA)

Tel. +1 217265 0886, E-Mail robmotl@illinois.edu 
activity is common in MS [9] and has been identified as a possible risk factor for cardiovascular comorbidities in this $[3,4]$ and the general population [10]. There is evidence of a linear, inverse association between physical activity and cardiovascular comorbidities, both self-reported and cases, in the general population [11] that has not yet been examined in persons with MS. Importantly, the generally high rate of physical inactivity in MS might reduce the possibility of an association of physical activity with cardiovascular comorbidities. This would indicate that other variables are possibly more important risk factors for cardiovascular comorbidities in MS. The present study examined the cross-sectional association between objectively measured and self-reported physical activity with self-reported cardiovascular comorbidities in persons with MS. We opted for a cross-sectional design to provide initial evidence on the existence and strength of association that could then support a prospective study of physical activity as a risk factor for cardiovascular disease (CVD) in MS. The hypothesis was of an inverse, linear association between level of physical activity and number of self-reported cardiovascular comorbidities even after accounting for disability status, sex, age, race, education, income, MS duration, and clinical course of MS as possible confounding variables.

\section{Methods}

\section{Participants}

The sample for this paper included 561 persons with MS who provided baseline data as part of two separate studies $[12,13]$ on physical activity and quality of life $(\mathrm{n}=292)$ and symptoms and physical activity $(n=269)$, respectively. The sample consisted of 468 women and 93 men (5:1 ratio), and 502 were diagnosed with relapsing-remitting MS and 59 were diagnosed with primary or secondary progressive MS. The mean age of the sample was 47.0 years $(S D=10.1$, range $=19-84$ years $)$ and the mean duration since definite diagnosis of MS was 9.6 years $(\mathrm{SD}=7.5$, range $=1-37$ years). The sample was mostly Caucasian (92\%) and educated ( $26 \%$ had some college education and $58 \%$ were college graduates) with a median annual household income of greater than USD $40,000(66 \%)$.

\section{Measures}

Physical Activity. Physical activity was measured by the Godin Leisure-Time Exercise Questionnaire (GLTEQ) [14] and the ActiGraph single-axis accelerometer (model 7164 version; Manufacturing Technology Incorporated, Fort Walton Beach, Fla., USA). The use of both self-report and objective measures is considered ideal [15] and allows for a partial examination of reporting bias in the analysis of physical activity and self-reported cardiovascular comorbidities. This addresses the concern that an association is just an artifact of using self-report as the only mode of data col- lection, but it does not address other possible information biases such as persons who are physically inactive self-reporting more cardiovascular symptoms without having CVD. Poor measurement as opposed to biased responses creates misclassification bias, which works against finding associations by biasing associations towards the null. The GLTEQ is a self-administered 2-part measure of usual physical activity; we only included the first part in this study consistent with previous research $[16,17]$. The first part has 3 items that measure the frequency of strenuous (e.g. jogging), moderate (e.g. fast walking), and mild (e.g. easy walking) exercise for periods of more than 15 min during one's free time in a typical week. The weekly frequencies of strenuous, moderate, and mild activities are multiplied by 9,5 , and 3 metabolic equivalents (METs), respectively, and summed to form a measure of total leisure activity that ranges between 0 and $119 \mathrm{MET}$ min/week. Physical inactivity is reflected by lower scores on the GLTEQ, whereas higher scores reflect greater physical activity levels.

The ActiGraph accelerometer contains a single, vertical-axis piezoelectric bender element that generates an electrical signal proportional to the force acting on it. The acceleration/deceleration signal is digitized by an analog-to-digital converter and numerically integrated over a preprogrammed epoch interval. At the end of each interval, the integrated value of movement counts is stored in RAM and the integrator is reset. The monitor is programmed for start time and data collection interval and data are retrieved for analysis via a PC interface and software provided with the unit. The downloaded data from the accelerometers are then entered into Microsoft Excel for data processing. In this study, the epoch was $1 \mathrm{~min}$, and the accelerometers were worn during the waking hours, except while showering, bathing, and swimming, for a 7-day period. Waking hours was defined as the moment upon getting out of bed in the morning through the moment of getting into bed in the evening. The accelerometers were not worn during the night while the participants slept. The participants recorded the time that the accelerometer was worn on a $\log$, and this was verified by inspection of the minute-by-minute accelerometer data. Regarding data processing, we summed the minute-by-minute counts across each of the 7 days and then averaged the total daily movement counts across the 7 days. This yielded accelerometer data in total movement counts per day. The lower bound score is 0 counts per day, whereas there is no upper boundary for accelerometer counts per day. Physical inactivity is reflected by lower movement counts per day, whereas higher scores reflect greater levels of physical activity. Both physical activity measures and the same procedures have been included in previous research on validity of physical activity measures and correlates/consequences of physical activity in MS patients [12, $13,16,17]$.

Self-Reported Cardiovascular Comorbidities. The measure of cardiovascular comorbidities involved a self-reported questionnaire that we developed based on the American College of Sports Medicine guidelines for measuring signs and symptoms suggestive of cardiovascular, pulmonary, or metabolic diseases [18, 19]. The questionnaire contained 9 items that participants rated 'yes' (1) or 'no' (0) based on experiencing the sign or symptom. Example items are 'Pain or discomfort (angina) in the chest, neck, jaw, arms or other areas that may be related to poor circulation (ischemia)', 'Heartbeats or palpitations that feel more frequent or forceful than usual or feeling that your heart is beating rapidly', 'Shortness of breath at rest or with mild exertion', 'Ankle swelling 
(edema) unrelated to injury', and 'A known heart murmur'. Similar self-administered questionnaires have been developed by researchers across North America and included as an initial, minimal step in the pre-exercise screening for cardiovascular conditions that are contraindications for exercise and exercise testing [18]. This practice provides a simple, fast, and acceptable screening whereby a greater number of signs or symptoms are indicative of greater risk for cardiovascular contraindications. The overall measure of self-reported cardiovascular comorbidities was generated by summing the scores from all 9 items into a single composite ranging between 0 and 9 , and higher scores indicated a greater number of self-reported cardiovascular comorbidities. Using data from the current sample, the internal consistency of this scale was adequate based on Cronbach's coefficient alpha $(\alpha=0.74)$. Similarly, preliminary evidence of validity, using data from the current sample, is based on a significant difference in mean scores between persons with and without family history of heart disease $(\mathrm{t}=2.61, \mathrm{~d}=0.29)$ and stroke $(\mathrm{t}=2.94, \mathrm{~d}=0.36)$, but not diabetes $(\mathrm{t}=0.77, \mathrm{~d}=0.09)$. Using another sample of unpublished data $(\mathrm{n}=$ 276), this scale has adequate test-retest reliability over a 6-month period based on an intraclass correlation coefficient of 0.70 and Spearman rho correlation coefficient $(\rho)$ of 0.66 . Additionally, scores from the scale were more strongly (Fisher's $\mathrm{z}=1.63, \mathrm{p}=$ $0.05)$ associated with self-reported physical health status $(\mathrm{r}=0.43$, $\rho=0.42)$ than mental health status $(r=0.31, \rho=0.29)$, as measured by SF-36. Collectively, this provides initial evidence for the reliability and validity of scores from the measures of self-reported cardiovascular comorbibities in MS.

Disability Status. Disability was measured using the PatientDetermined Disease Steps (PDDS) scale [20]. The PDDS scale is a self-report questionnaire that contains a single item for measuring self-reported disability using an 8-level ordinal scale. The description for 0 was 'normal' or 'I may have some mild symptoms, mostly sensory due to MS but they do not limit my activity. If I do have an attack, I return to normal when the attack has passed'. The description for 4 was 'early cane' or 'I use a cane or a single crutch or some other form of support (such as touching a wall or leaning on someone's arm) for walking all the time or part of the time, especially when walking outside. I think I can walk 25 feet in $20 \mathrm{~s}$ without a cane or crutch. I always need some assistance (cane or crutch) if I want to walk as far as 3 blocks'. The description for 8 was 'bedridden' or 'Unable to sit in a wheelchair for more than one hour'. This scale was developed as an inexpensive surrogate for the Expanded Disability Status Scale and scores from the PDDS scale are linearly and strongly related with physicianadministered Expanded Disability Status Scale scores $(r=0.93)$ [20] thereby providing evidence for the validity of this scale as a measure of disability.

Demographic/Clinical Information. The demographic information of sex, age, race, education, and income were collected using a self-report scale. The confirmed diagnosis, duration of MS, and clinical course of MS were collected from the patient's neurologist using a standard form letter.

\section{Procedures}

The procedures for both studies were approved by a university institutional review board. After initial telephone contact, screening for inclusion, and return of written informed consent and MS verification documentation, participants were sent an accelerometer and battery of questionnaires through the United States Post- al Service for baseline, cross-sectional data collection. We further provided prestamped and preaddressed envelopes for return. The project coordinator called to make sure the participants received the materials and understood the instructions. The participants then completed the battery of baseline questionnaires that included demographic, cardiovascular comorbidities, neurological impairment, and physical activity assessments, and then wore the accelerometer for 7 days. After completing the assessments and wearing the accelerometer, participants returned the study materials through the United States Postal Service. We contacted participants by telephone and e-mail up to 3 times as a reminder to return the study materials. We further collected any missing questionnaire data based on up to 3 times follow-up telephone calls. All participants received USD 20 remuneration upon returning the study materials.

\section{Data Analysis}

All analyses were performed in PASW 18.0. We initially performed descriptive and distributional analyses on the primary outcomes of self-reported cardiovascular comorbidities and physical activity as well as disability status. The next analysis involved an estimation of parametric (r) and nonparametric $(\rho)$ bivariate correlations between number of self-reported cardiovascular comorbidities, physical activity, and the potential confounding variables of disability status (PDDS scores), sex $(0=$ female, 1 = male), age (years), race $(0=$ Caucasian, $1=$ other $)$, education $(0=$ high school or less, $1=$ some college, $2=$ college graduate), income ( $0=\leq 40,000 \mathrm{USD} /$ year, $1=>40,000 \mathrm{USD} /$ year), MS duration (years), and clinical course of MS ( $0=$ relapsing-remitting, 1 = progressive). The final analysis involved multiple linear regression models. We regressed the number of selfreported cardiovascular comorbidities on confounding variables in block 1 and then entered physical activity, either GLTEQ or accelerometry, in block 2 . We examined the significance of the change in the $\mathrm{R}^{2}$ value between the two blocks for judging the independent contribution of physical activity for explaining variance in the number of self-reported cardiovascular comorbidities beyond that of the confounding variables. This analysis was performed separately for the GLTEQ and accelerometer as measures of physical activity. We adopted a conservative $\mathrm{p}$ value of 0.01 for judging the statistical significance in all analyses given the large number of tests in the bivariate and regression analyses. We converted the standardized $\beta$ coefficient from the multiple linear regression into an odds ratio (OR) using the cosine-pi formula for a tetrachoric correlation taken from Ferguson [21] for simple interpretation within an epidemiological framework. We did not conduct logistic regression because of the evidence of a linear, inverse association between physical activity and CVD in the general population [11].

\section{Results}

Descriptive and Distributional Analyses for Primary Variables

The means \pm standard deviation for the self-reported cardiovascular comorbidities, GLTEQ, and accelerometer were $2.5 \pm 2.2$ comorbidities (range $=0-9$ ), $27.1 \pm$ 
Table 1. Bivariate correlations among cardiovascular comorbidity score, physical activity, and confounding variables

\begin{tabular}{lcccccccccccc}
\hline Variable & 1 & 2 & 3 & 4 & 5 & 6 & 7 & 8 & 9 & 10 & 11 \\
\hline 1 Cardiovascular comorbidities & - & $-0.225^{*}$ & $-0.161^{*}$ & $0.239^{*}$ & -0.033 & 0.039 & 0.026 & $-0.110^{*}$ & -0.030 & -0.008 & -0.026 \\
2 Accelerometer & $-0.192^{*}$ & - & $0.385^{*}$ & $-0.423^{*}$ & 0.037 & $-0.146^{*}$ & -0.030 & 0.101 & 0.032 & -0.067 & $-0.165^{*}$ \\
3 GLTEQ & $-0.151^{*}$ & $0.383^{*}$ & - & $-0.263^{*}$ & -0.012 & $-0.123^{*}$ & 0.059 & 0.023 & -0.001 & -0.016 & $-0.109^{*}$ \\
4 PDDS & $0.222^{*}$ & $-0.389^{*}$ & $-0.264^{*}$ & - & 0.058 & $0.167^{*}$ & -0.073 & -0.044 & -0.005 & 0.098 & $0.230^{*}$ \\
5 Sex & -0.033 & 0.028 & -0.018 & 0.057 & - & -0.077 & 0.034 & 0.069 & -0.017 & -0.098 & 0.035 \\
6 Age & 0.031 & $-0.177^{*}$ & -0.102 & 0.180 & -0.085 & - & $-0.110^{*}$ & -0.050 & 0.042 & $0.414^{*}$ & $0.187^{*}$ \\
7 Race & 0.044 & -0.018 & 0.071 & -0.074 & 0.034 & -0.109 & - & -0.001 & $-0.156^{*}$ & -0.040 & 0.076 \\
8 Education & -0.094 & 0.083 & 0.017 & -0.040 & 0.067 & -0.039 & 0.001 & - & $0.167^{*}$ & 0.040 & 0.018 \\
9 Income & -0.029 & -0.008 & -0.012 & -0.005 & -0.017 & 0.069 & $-0.156^{*}$ & $0.157^{*}$ & - & 0.052 & -0.044 \\
10 Disease duration & -0.018 & -0.061 & -0.008 & 0.070 & -0.070 & $0.403^{*}$ & -0.061 & 0.017 & 0.054 & - \\
11 MS clinical course & -0.028 & $-0.152^{*}$ & -0.105 & $0.241^{*}$ & 0.035 & $0.200^{*}$ & 0.076 & 0.018 & -0.044 & $0.233^{*}$ & - \\
\hline
\end{tabular}

Values below the diagonal are parametric correlations, whereas values above the diagonal are nonparametric correlations. Cardiovascular comorbidities are self-reported major signs and symptoms rather than cases of CVD. ${ }^{*} \mathrm{p}<0.01$, two-tailed.

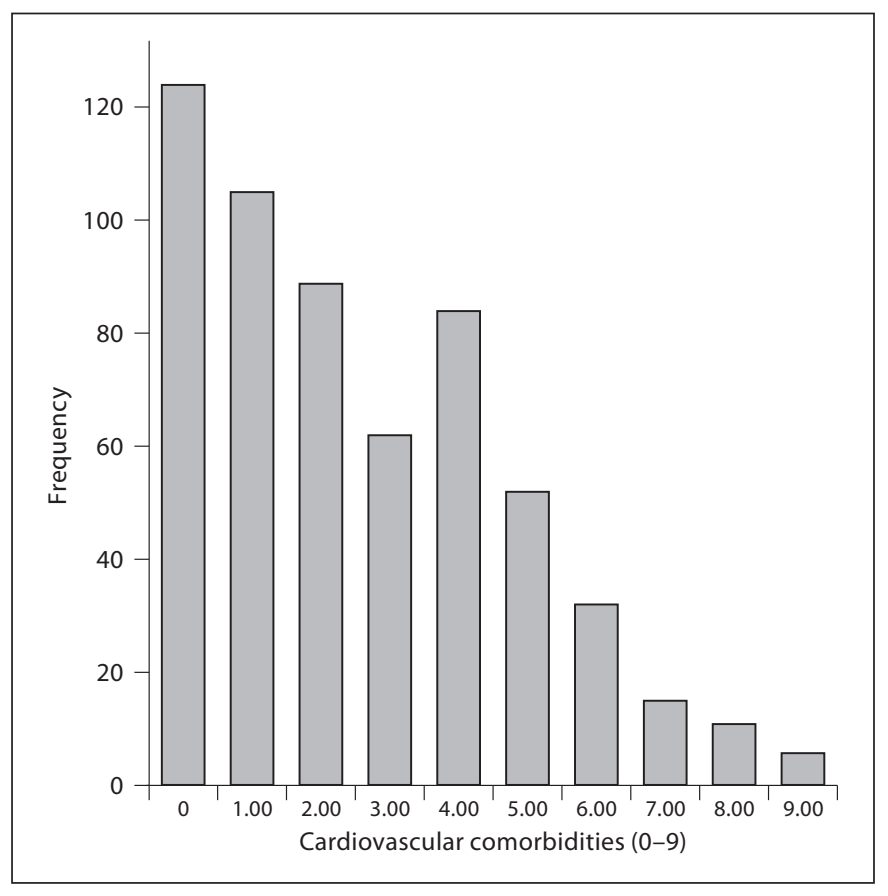

Fig. 1. Frequency distribution for the number of self-reported cardiovascular comorbidities in the sample of persons with MS.

22.6 $\mathrm{MET} \mathrm{min} /$ week (range $=0-119)$, and 215,812 \pm 110,508 counts per day (range $=18,929-760,722$ ), respectively. The histogram in figure 1 indicates that self-reported cardiovascular comorbidities were common in this sample of persons with MS as only $22 \%$ or 123 participants self-reported no cardiovascular comorbidities. The mean values for the GLTEQ and accelerometer are consistent with previous research involving persons with MS $[16,17]$ indicating that that sample was generally physically inactive. The median PDDS score was 2 (range $=0-6$ ) indicating moderate disability [20]. The estimates of skewness were $0.66,1.06$, and 1.63 for the selfreported cardiovascular comorbidity score, GLTEQ, and accelerometer, respectively. The estimates of kurtosis were $-0.30,1.15$, and 4.36 for the cardiovascular comorbidity score, GLTEQ, and accelerometer, respectively. There were $7 \%$ of missing accelerometer data, and less than $1 \%$ of missing cardiovascular comorbidity and GLTEQ data.

\section{Bivariate Correlations}

The bivariate correlations are provided in table 1, and, notably, there were statistically significant negative correlations between the self-reported cardiovascular comorbidity score and accelerometer $(r=-0.192, p=0.0001$; $\rho=-0.225, \mathrm{p}=0.0001)$ and GLTEQ $(\mathrm{r}=-0.151, \mathrm{p}=0.0001$; $\rho=-0.161, p=0.0001)$ scores. This indicates that those with MS who were less physically active self-reported a greater number of cardiovascular comorbidities. We further note that PDDS scores were positively correlated with the self-reported cardiovascular comorbidity score $(\mathrm{r}=0.222, \mathrm{p}=0.0001 ; \rho=0.239, \mathrm{p}=0.0001)$ and negatively correlated with accelerometer $(\mathrm{r}=-0.389, \mathrm{p}=$ $0.0001 ; \rho=-0.423, \mathrm{p}=0.0001)$ and GLTEQ $(\mathrm{r}=-0.264$, $\mathrm{p}=0.0001 ; \rho=-0.263, \mathrm{p}=0.0001)$ scores. This indicates that those with MS who reported greater disability further self-reported a greater number of cardiovascular comorbidities and were less physically active.

\section{Multiple Linear Regression Analyses}

The first regression analysis examined the association between accelerometer scores (i.e., objectively measured 
Table 2. Summary of multiple linear regression analysis for selfreported cardiovascular comorbidities using objectively measured physical activity

\begin{tabular}{lrrr}
\hline Variable & \multicolumn{1}{c}{ B } & SE B & \multicolumn{1}{c}{$\beta$} \\
\hline Block 1 & & & \\
PDDS & 0.324 & 0.058 & $0.252^{*}$ \\
Sex & -0.252 & 0.248 & -0.044 \\
Age & 0.002 & 0.010 & 0.011 \\
Race & 0.562 & 0.371 & 0.067 \\
Education & -0.231 & 0.126 & -0.081 \\
Income & -0.085 & 0.205 & -0.018 \\
Disease duration & 0.002 & 0.014 & 0.005 \\
MS clinical course & -0.615 & 0.325 & -0.087 \\
Block 2 & & & \\
Accelerometer & -0.003 & 0.001 & $-0.128^{*}$ \\
PDDS & 0.262 & 0.062 & $0.203^{*}$ \\
Sex & -0.237 & 0.246 & -0.042 \\
Age & 0.001 & 0.010 & 0.000 \\
Race & 0.508 & 0.369 & 0.060 \\
Education & -0.201 & 0.126 & -0.070 \\
Income & -0.105 & 0.203 & -0.023 \\
Disease duration & 0.003 & 0.014 & 0.003 \\
MS clinical course & -0.664 & 0.324 & -0.094 \\
\hline
\end{tabular}

$\mathrm{R}^{2}=0.074$ for block $1 ; \Delta \mathrm{R}^{2}=0.013$ for block $2(\mathrm{p}=0.007)$. ${ }^{*} \mathrm{p}<0.01$, two-tailed.
Table 3. Summary of multiple linear regression analysis for selfreported cardiovascular comorbidities using self-reported physical activity

\begin{tabular}{lrrr}
\hline Variable & B & SE B & $\beta$ \\
\hline Block 1 & & & \\
PDDS & 0.309 & 0.055 & $0.248^{*}$ \\
Sex & -0.243 & 0.242 & -0.042 \\
Age & 0.003 & 0.010 & 0.014 \\
Race & 0.569 & 0.344 & 0.071 \\
Education & -0.191 & 0.123 & -0.066 \\
Income & -0.058 & 0.198 & -0.013 \\
Disease duration & -0.004 & 0.013 & -0.016 \\
MS clinical course & -0.612 & 0.311 & -0.088 \\
Block 2 & & & \\
GLTEQ & -0.011 & 0.004 & $-0.114^{*}$ \\
PDDS & 0.274 & 0.056 & $0.220^{*}$ \\
Sex & -0.261 & 0.240 & -0.045 \\
Age & 0.002 & 0.010 & 0.010 \\
Race & 0.624 & 0.343 & 0.078 \\
Education & -0.186 & 0.122 & -0.064 \\
Income & -0.063 & 0.197 & -0.014 \\
Disease duration & -0.003 & 0.013 & -0.011 \\
MS clinical course & -0.653 & 0.310 & -0.094 \\
\hline
\end{tabular}

$\mathrm{R}^{2}=0.069$ for block $1 ; \Delta \mathrm{R}^{2}=0.012$ for block $2(\mathrm{p}=0.009) ;$ ${ }^{*} \mathrm{p}<0.01$, two-tailed. physical activity) and self-reported cardiovascular comorbidity scores controlling for confounding variables, and the results are provided in table 2 . All of the confounding variables were entered in block 1 and explained a significant portion of variance in self-reported cardiovascular comorbidity scores $[\mathrm{F}(8,498)=4.98, \mathrm{p}=0.001$, $\left.\mathrm{R}^{2}=0.074\right]$. Of the confounding variables, only PDDS scores (i.e., disability status) were significantly correlated with self-reported cardiovascular comorbidity scores $(B=0.324$, SE $B=0.058, \beta=0.252)$; this standardized $\beta$ coefficient translates into an OR of 1.92. Accelerometer scores were added in block 2 and explained significantly and incrementally more variance in self-reported cardiovascular comorbidity scores $[\mathrm{F}(1,497)=7.348, \mathrm{p}=0.007$, $\left.\mathrm{R}^{2}=0.013\right]$. Both accelerometer $(\mathrm{B}=-0.003$, $\mathrm{SE} \mathrm{B}=0.001$, $\beta=-0.128)$ and PDDS $(B=0.262, \operatorname{SE} B=0.062, \beta=0.203)$ scores were significantly associated with self-reported cardiovascular comorbidity scores. Those who were less physically active and reported worse disability status selfreported a higher number of cardiovascular comorbidities, and the partial regression plot along with lines of best fit and 95\% confidence intervals for the association between physical activity and self-reported cardiovascular comorbidities is presented in figure 2 . The coefficients for accelerometer counts and PDDS scores (i.e., disability status) translated into ORs of 1.4 and 1.7 , respectively.

The second regression analysis examined the association between GLTEQ (i.e., self-reported physical activity) and self-reported cardiovascular comorbidity scores controlling for confounding variables and the results are provided in table 3. All of the confounding variables were again entered in block 1 and explained a significant portion of variance in self-reported cardiovascular comorbidity scores $\left[\mathrm{F}(8,534)=4.94, \mathrm{p}=0.001, \mathrm{R}^{2}=0.069\right]$. Of the variables, only PDDS scores (i.e., disability status) were significantly correlated with self-reported cardiovascular comorbidity scores $(B=0.309, S E B=0.055$, $\beta=0.248$ ); this standardized $\beta$ coefficient translates into an OR of 1.90. GLTEQ scores were added in block 2 and explained significantly and incrementally more variance in self-reported cardiovascular comorbidity scores $\left[F(1,533)=6.97, p=0.009, R^{2}=0.012\right]$, and both GLTEQ $(\mathrm{B}=-0.011, \mathrm{SE} \mathrm{B}=0.004, \beta=-0.114)$ and PDDS $(\mathrm{B}=$ 0.274 , SE $B=0.056, \beta=0.220$ ) scores were significantly 


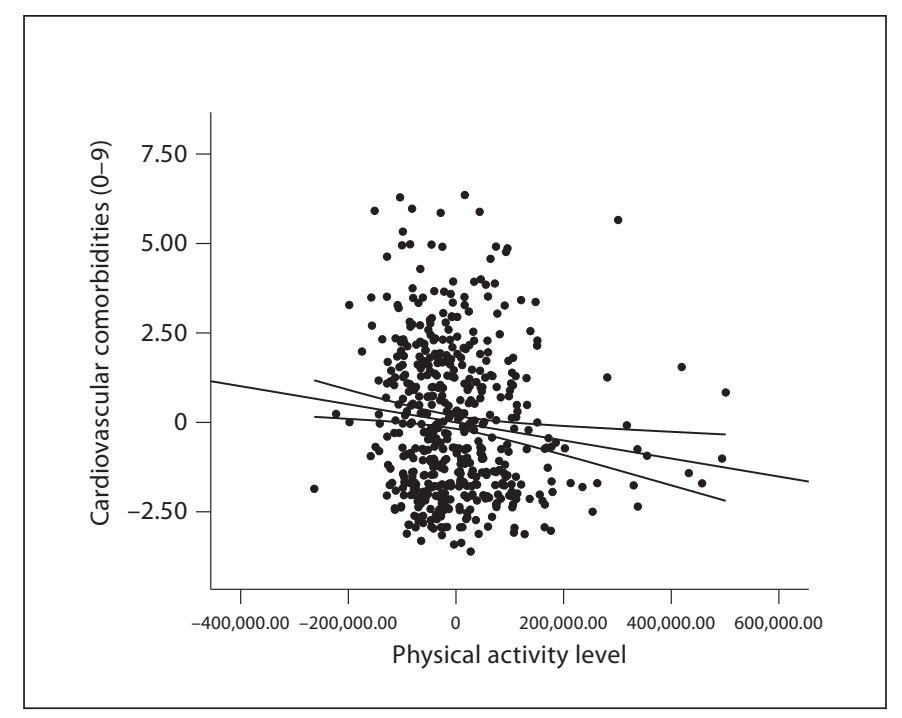

Fig. 2. Partial regression plot along with lines of best fit and $95 \%$ confidence intervals for the association between objectively measured physical activity and number of self-reported cardiovascular comorbidities adjusting for disability, MS clinical course, MS duration, sex, age, race, education, and income.

associated with self-reported cardiovascular comorbidity scores. Those who reported being less physically active and having worse disability status self-reported a higher number of cardiovascular comorbidities; the partial regression plot along with lines of best fit and 95\% confidence intervals for the association between physical activity and self-reported cardiovascular comorbidities is presented in figure 3. The coefficients for GLTEQ and PDDS scores (i.e., disability status) translated into ORs of 1.3 and 1.8 , respectively.

\section{Discussion}

The primary contribution of the present cross-sectional analysis was the observation that physical activity was linearly and inversely associated with the number of self-reported cardiovascular comorbidities, independent of disability status and other possible confounding variables including age and gender, in persons with MS. Such an analysis was motivated by observations of an increased risk of cardiovascular comorbidities $[3,4]$ and an elevated prevalence of physical inactivity [9] in persons with MS. Furthermore, there is convincing evidence from the general population without MS of an association between physical activity and self-reported and cases of cardiovas-

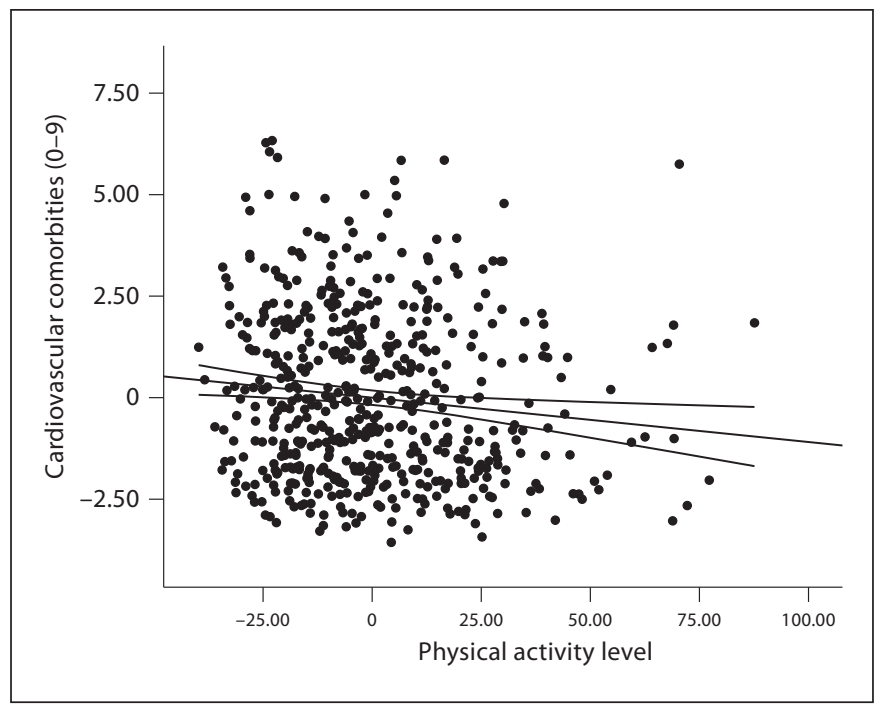

Fig. 3. Partial regression plot along with lines of best fit and $95 \%$ confidence intervals for the association between self-reported physical activity and number of self-reported cardiovascular comorbidities adjusting for disability, MS clinical course, MS duration, sex, age, race, education, and income.

cular comorbidities [10] that is inverse and linear [11]. To that end, we now provide novel evidence that physical activity is inversely and independently associated with selfreported cardiovascular comorbidities in persons with MS. The importance of such an observation is that physical activity is potentially modifiable by a well-designed behavioral intervention in persons with MS and even small changes in physical activity among those with and without ambulation impairments might result in a reduced risk of cardiovascular comorbidities from a public health perspective. The promotion of physical activity in $\mathrm{MS}$ is an area ripe for future inquiry as it is a primary behavioral factor associated with a number of positive consequences in MS, and our data would add reduced number of self-reported cardiovascular comorbidities to the list of possible desirable outcomes of this behavior. With improvements in drug therapy for MS, added self help efforts may further improve quality of life for MS patients and reduce the risks of future CVD.

Herein, we reported standardized $\beta$ coefficients for the association between physical activity and number of self-reported cardiovascular comorbidities that were converted into ORs of 1.3 and 1.4 for the self-report and objective measures of physical activity, respectively. The magnitude of the associations between physical activity and self-reported cardiovascular comorbidities is less 
than that of disability status (i.e., ORs $=1.7-1.8$ ), but the nature of the association is consistent with much of the research on physical activity and cardiovascular comorbidities, both self-reported and cases, in the general population $[10,11]$. For example, the risks of coronary heart and cardiovascular diseases decreased linearly in association with increasing percentiles of physical activity in a meta-analysis of epidemiological studies published before and after the Surgeon General's report on physical activity and health [11]. The consistency of the linear, inverse association between physical activity and cardiovascular comorbidities in both MS and the general population might be surprising given that persons with MS are considerably less physically active than the general population [6] and this might undermine the parametric, linear analysis based on a truncated distribution, particularly considering the prevalence of physical inactivity. Nevertheless, we observed a cross-sectional association between physical activity and number of self-reported cardiovascular comorbidities even in a generally physically inactive population and such a result provides initial evidence that even small increases in physical activity might be associated with a reduction in cardiovascular comorbidities in MS as in the general population.

Although not a direct purpose of our research, we reported an association between disability status and selfreported cardiovascular comorbidities in persons with MS. The standardized $\beta$ coefficient for the association approached an OR of 2.0, was independent of other confounding variables and physical activity, and was consistent in magnitude with that reported by others using selfreports of disability and comorbidities [22]. This indicates that disability status is associated with an increase in self-reported cardiovascular comorbidities and other self-reported comorbid conditions in MS, although we do recognize that cardiovascular comorbidities might be a risk factor for disability. Interestingly, the association between disability status and self-reported cardiovascular comorbidities was not attenuated in the analysis that included physical activity, thereby indicating that the physical activity is not likely influencing cardiovascular comorbidities through a reduction in physical disability, although such a possibility could be further examined in prospective, longitudinal studies. We further did not examine the possibility of associations between physical activity and number of self-reported cardiovascular comorbidities within groups that differed in disability status; there were too few persons classified with either high physical activity in the moderate disability group or low physical activity in mild disability group in our follow-up univariate ANOVAs.

An important and novel feature of this study was the inclusion of both self-report and objective measures of physical activity, and the observation of similar associations between both types of physical activity measures with the number of cardiovascular comorbidities in MS. The inclusion of two types of measures is not commonplace in epidemiological studies of physical activity and morbidity. However, our data address the concern that an association between physical activity and cardiovascular comorbidities reflects a bias of only using self-reported assessments (i.e., self-report method bias). If the association between physical activity and cardiovascular comorbidities was a product of this type of bias, then one would expect differential associations between objectively measured and self-reported physical activity and number of self-reported cardiovascular comorbidities. This was not observed in the present analysis and further strengthens the notion that the association between physical activity and cardiovascular comorbidities in persons with MS reflects a biologically plausible process. Nevertheless, our design does not address another type of information bias whereby less physically active persons report cardiovascular signs and symptoms, without having CVD. If reporting biases are not differential by physical activity levels, the misclassification would bias the correlations toward 0 and make the results of our findings conservative.

There is a plausible biological basis for our finding of an association between physical activity and self-reported cardiovascular comorbidities in MS. Chronic inflammation is a predictor of CVD morbidity and mortality and is further involved in atherosclerotic plaque formation. Inflammation further decreases endothelial function and increases arterial stiffness [23-25], and arterial dysfunction is present long before measureable atherosclerotic lesions and CVD signs and symptoms [23]. These changes in arterial function coupled with greater intima-media thickness provide evidence for early subclinical atherosclerosis and are prominent in other autoimmune diseases [26-28], but little information exists in populations with MS. By extension, physical activity might be associated with decreased systemic inflammation $[29,30]$ and, in turn, be protective against the development of cardiovascular comorbidities in MS by reducing subclinical atherosclerosis, even in the absence of overt CVD symptoms. Since the major class of MS therapies, such as interferons, is aimed at reducing inflammation, but not approved as therapies for secondary progres- 
sive or progressive MS, such adjuncts as physical activity may be important.

One practical implication of our results is that physical activity in a potentially modifiable behavior that, if successfully altered, might reduce cardiovascular comorbidities as well as promote other desirable consequences in MS. Indeed, physical activity in the form of exercise training has been associated with improvements in symptoms, mobility, and quality of life in MS [31-33], and further research might examine the effects of exercise training on subclinical markers of atherosclerosis as early predictors of subsequent cardiovascular comorbidities in MS. We further note that researchers might consider the promotion of lifestyle physical activity behavior as an approach for reducing cardiovascular comorbidities and other comorbidities in MS.

The strengths of the current study include the focus on physical activity as a potentially alterable behavior that is associated with cardiovascular comorbidities in MS using two measures of physical activity and control for disability status and other confounding variables. The present study is not without major limitations. Importantly, the cross-sectional design did not establish the temporal sequence between physical activity and self-reported cardiovascular comorbidities, and the observed association could reflect pre-existing conditions and/or an effect of physical activity on cardiovascular comorbidities or of cardiovascular comorbidities on physical activity. Essentially, we are unable to fully establish physical activity as a risk factor for CVD, but rather set the stage for such a prospective examination in persons with MS. The outcome was a self-reported measure of cardiovascular comorbidities based on items from a questionnaire that we designed using American College of Sports Medicine screening criteria for major signs and symptoms of CVD rather than actual cases. The inclusion of a self-report measure of cardiovascular comorbidities was based on evidence that questionnaires can provide a reliable, valid, and practical alternative for medical record abstraction, a technique that can be costly and impractical in research and biased by the accuracy, purpose (billing), and thoroughness of documentation in medical records $[34,35]$. We further note that research participants can reliably and accurately report medical conditions in epidemiological studies, particularly those that are well-defined and serious such as major signs and symptoms of CVD $[34,35]$. Nevertheless, we provided initial evidence that the scores from the self-reported measure of cardiovascular comorbidities in this study were reliable, stable over time, demonstrated mean differences based on family history of CVD (i.e., heart disease and stroke), and correlated in a predictable manner with self-reported physical and mental health status. We do recognize that our analyses should be replicated using objective measures of CVD as an outcome. Lastly, the sample was relatively small and did not include controls without MS for a direct comparison of generalizability or specificity of the association. The sample further had a ratio of sex and disease classification that are not perfectly representative of people with MS, but the reported ratios of 2 to 3 to 4 females to 1 male are consistently reported in the literature. To that end, future researchers might consider a prospective, observational study for further examining physical activity as a risk factor for CVD events (i.e., cases) in a more representative sample of persons with MS and controls.

Collectively, we provide novel cross-sectional evidence that physical activity is inversely and independently associated with self-reported cardiovascular comorbidities in persons with MS. There are significant limitations of this initial study, and this line of research should be extended into prospective, longitudinal examinations of physical activity and cases of CVD as well as laboratory-based examinations of the association between physical activity and subclinical atherosclerosis, even in the absence of overt CVD symptoms, in persons with MS.

\section{Acknowledgements}

This study was funded by the National Institute of Neurological Diseases and Stroke (NS054050) and the National Multiple Sclerosis Society (RG 3926A2/1).

References Incidence and prevalence of multiple sclerosis in Olmsted County, Minnesota, 19852000. Neurology 2003;61:1373-1377.

2 Marrie RA, Horwitz R, Cutter G, et al: Comorbidity, socioeconomic status and multiple sclerosis. Mult Scler 2008;14:1091-1098.

-3 Christiansen CF, Christensen S, Farkas DK, et al: Risk of arterial cardiovascular disease in patients with multiple sclerosis: a population-based cohort study. Neuroepidemiology 2010;35:267-274.

4 Kang JH, Chen YH, Lin HC: Comorbidities among patients with multiple sclerosis: a population-based controlled study. Eur J Neurol 2010;17:1215-1219.

5 Marrie RA, Rudick R, Horwitz R, et al: Vascular comorbidity in associated with more rapid disability progression in multiple sclerosis. Neurology 2010;74:1041-1047. 
6 Koch-Henriksen N, Bronnum-Hansen H, Stenager E: Underlying cause of death in Danish patients with multiple sclerosis: results from the Danish Multiple Sclerosis Registry. J Neurol Neurosurg Psychiatry 1998;65:56-59.

$\checkmark 7$ Grytten Torkildsen N, Lie SA, Aarseth JH, Nyland H, Myhr KM: Survival and cause of death in multiple sclerosis: results from a 50year follow-up in Western Norway. Mult Scler 2008;14:1191-1198.

$\checkmark 8$ Hirst C, Swingler R, Compston DAS, BenShlomo Y, Robertson NP: Survival and cause of death in multiple sclerosis: a prospective population-based study. J Neurol Neurosurg Psychiatry 2008;79:1016-1021.

9 Motl RW, McAuley E, Snook EM: Physical activity and multiple sclerosis: a meta-analysis. Mult Scler 2005;11:459-463.

10 Physical activity and cardiovascular health. NIH Consensus Development Panel on Physical Activity and Cardiovascular Health. JAMA 1996;276:241-246.

11 Williams PT: Physical fitness and activity as separate heart disease risk factors: a metaanalysis. Med Sci Sports Exerc 2001;33:754761.

-12 Motl RW, McAuley E, Snook EM, et al: Physical activity and quality of life in multiple sclerosis: intermediary roles of disability, fatigue, mood, pain, self-efficacy, and social support. Psychol Health Med 2009;14:111124.

-13 Motl RW, McAuley E, Wynn D, et al: Symptoms and physical activity among adults with relapsing-remitting multiple sclerosis? J Nerv Ment Dis 2010;198:213-219.

14 Godin G, Shephard RJ: A simple method to assess exercise behavior in the community. Can J Appl Sport Sci 1985;10:141-146.

- 15 Dishman RK, Washburn RA, Schoeller DA: Measurement of physical activity. Quest 2001;53:295-309.
16 Gosney JL, Scott JA, Snook EM, et al: Physical activity and multiple sclerosis: validity of self-report and objective measures. Fam Commun Health 2007;30:144-150.

17 Motl RW, McAuley E, Snook EM, et al: Validity of physical activity measures in ambulatory individuals with multiple sclerosis. Disabil Rehabil 2006;28:1151-1156.

18 American College of Sports Medicine: ACSM's Guidelines for Exercise Testing and Prescription, ed 7. Baltimore, Lippincott, Williams, \& Wilkins, 2005.

19 Gordon S, Mitchell BS: Health appraisal in the non-medical setting; in Durstine JL, King AC, Painter PL (eds): ACSM's Resource Manual for Guidelines for Exercise Testing and Prescription. Philadelphia, Lea \& Febinger, 1993, pp 219-228.

20 Hadjimichael O, Kerns RB, Rizzo MA, et al: Persistent pain and uncomfortable sensations in persons with multiple sclerosis. Pain 2007; 127:35-41.

21 Ferguson GA: Statistical Analysis in Psychology and Education. New York, McGrawHill, 1966, p 244.

22 Marrie RA, Horwitz R, Cutter G, et al: Comorbidity delays diagnosis and increase disability at diagnosis in MS. Neurology 2009; 72:117-124.

23 Abou-Raya A, Abou-Raya S: Inflammation a pivotal link between autoimmune diseases and atherosclerosis. Autoimmun Rev 2006; 5:331-337.

24 Agewall S: Is impaired flow-mediated dilatation of the brachial artery a cardiovascular risk factor? Curr Vasc Pharmacol 2003;1: 107-109.

25 Libby P: Inflammation in atherosclerosis. Nature 2002;420:868-874.
26 Van Doornum S, McColl G, Wicks IP: Atorvastatin reduces arterial stiffness in patients with rheumatoid arthritis. Ann Rheum Dis 2004;63:1571-1575.

27 Wong M, Toh L, Wilson A, et al: Reduced arterial elasticity in rheumatoid arthritis and the relationship to vascular disease risk factors and inflammation. Arthritis Rheum 2003;48:81-89.

28 Kumeda Y, Inaba M, Goto H, et al: Increased thickness of the arterial intima-media detected by ultrasonography in patients with rheumatoid arthritis. Arthritis Rheum 2002; 46:1489-1497.

29 Giannopoulou I, Fernhall B, Carhart R, et al: Effects of diet and/or exercise on the adipocytokine and inflammatory cytokine levels of postmenopausal women with type 2 diabetes. Metabolism 2005;54:866-875.

30 Jae SY, Fernhall B, Heffernan KS, et al: Chronotropic response to exercise testing is associated with carotid atherosclerosis in healthy middle-aged men. Eur Heart J 2006;27:954959.

-31 Garrett M, Coote S: Multiple sclerosis and exercise in people with minimal gait impairment - a review. Phys Ther Rev 2009;14:169180.

32 Motl RW, McAuley E, Snook EM: Physical activity and multiple sclerosis: a meta-analysis. Mult Scler 2005;11:459-463.

-33 Snook EM, Motl RW: Effect of exercise training on walking mobility in multiple sclerosis: a meta-analysis. Neurorehabil Neural Repair 2009;23:108-116

34 Katz JN, Chang LC, Sangha O, Fossel AH, Bates DW: Can comorbidity be measured by questionnaire rather than medical record review? Med Care 1996;34:73-84.

-35 Sangha O, Stucki G, Liang MH, Fossel AH, Katz JN: The Self-Administered Comorbidity Questionnaire: a new method to assess comorbidity for clinical and health services research. Arthritis Rheum 2003;49:156-163. 